\title{
Development of new software enabling automatic identification of the optimal anatomical liver resectable region, incorporating preoperative liver function
}

\author{
MITSUGI SHIMODA $^{1}$, MASANORI HARIYAMA ${ }^{1,2}$, YUKIO OSHIRO $^{1}$ and SHUJI SUZUKI ${ }^{1}$ \\ ${ }^{1}$ Department of Gastroenterological Surgery, Tokyo Medical University, Ibaraki Medical Center, Ibaraki 300-0395; \\ ${ }^{2}$ Graduate School of Information Sciences, Tohoku University, Sendai 980-8579, Japan
}

Received April 4, 2019; Accepted September 6, 2019

DOI: $10.3892 / 01.2019 .11006$

\begin{abstract}
Determining the resectable region and volume of the liver prior to anatomical resection is important. The synapse Vincent (SV) system is the current method for surgical liver resection that relies on the surgeon's individual experience and skill. Additionally, in cases involving abnormal liver function, the resectable region is limited due to deteriorating liver function, thus making the determination of the hepatectomy region challenging. The current study outlines a novel 3D Hariyama-Shimoda Soft (HSS) simulation software that can be used to automatically simulate the optimal hepatectomy region under a limited resectable liver volume. The current study recruited patients with hepatic malignant tumors that were scheduled for anatomical resection. The influence of the tumor on each portal vein point was quantified in accordance with the tumor domination ratio (TDR). The resectable region was subsequently determined so that the sum of the TDR was the maximum estimated resectable liver volume (ERLV). The maximum ERLV settings utilized were within Makuuchi's criteria. ERLV was compared with the actual resected liver volume (ARLV) using SV and HSS. A total of 15 patients were included in the present study. The median ERLV was not significantly different between the two groups $(\mathrm{P}=0.15)$. However, the correlation between ERLV and ARLV, for SV and HSS, was statistically significant $[\mathrm{SV} \operatorname{ERLV}(\mathrm{ml})=1.139 \times \mathrm{HSS}$ $\operatorname{ERLV}(\mathrm{ml})+30.779(\mathrm{P}=0.001)]$. In conclusion, HSS may be an effective 3D simulation system. TDR and ERLV were indicated to be novel factors that may be incorporated into simulation software for use in anatomical resection surgery.
\end{abstract}

Correspondence to: Dr Mitsugi Shimoda, Department of Gastroenterological Surgery, Tokyo Medical University, Ibaraki Medical Center, 3-20-1 Chuo, Ami, Ibaraki 300-0395, Japan E-mail:mshimoda@tokyo-med.ac.jp

Key words: anatomical resection, three dimensional CT simulation, estimated resectable liver volume

\section{Introduction}

Preoperative clinical imaging is an important diagnostic tool for malignant liver tumors. CT imaging has improved rapidly in recent years due to technological advancements. Currently, clinicians, and especially hepatobiliary surgeons, can use three-dimensional (3D) images that are visually easier to comprehend than conventional two-dimensional (2D) images. 3D imaging is especially useful in for the diagnosis of a variety of liver diseases. CT imaging is now used to plan surgery, visualise the vascular anatomy and tumor location, and to identify liver resection regions and estimated resectable liver volume (ERLV). The synapse Vincent (SV: Fujifilm Medical Co., Ltd.) is a $3 \mathrm{D}$ image analytical software that is commonly used worldwide to visualise the detailed vascular anatomy of the liver. The SV is widely used in Japan, with $>95 \%$ high volume centers using this system (1). SV has been used in all cases of liver resection from 2012. However, the SV system presents some limitations: i) Manual adjustments are necessary for regions with poor vascular visual clearance and these require a lot of time to configure; ii) controversy exists as to whether all portal veins feeding into the tumor are identified using this technique; iii) it is difficult to determine the resectable regions when the liver function is deteriorating and the SV system does not incorporate pre-operative liver function, including that based on the Child-Pugh score or the indocyanine green retention test rate 15 ( ICG R15); iv) each surgeon manually uses SV, so the results can vary depending on the skills of each individual.

To resolve the limitations of this software, the current study used the Hariyama-Shimoda Soft (HSS) simulation software, which incorporates an identification function of a $100 \%$ dominant portal vein from the tumor, and a pre-operative evaluation mechanism that incorporated the maximum ERLV simulated from Makuuchi's criteria (2). HSS is a fully automated 3D simulation software which was developed in 2011 (3). In the current study, the results of HSS with SV are compared to evaluate the efficacy of HSS as a 3D simulation software. Additionally, whether the HSS produced similar results to the SV software was also examined. 


\section{Materials and methods}

Patient selection. All 15 patients in the study population underwent curative resection and required anatomical liver resection for a single liver malignancy lesion at the Department of Gastroenterological Surgery, Ibaraki Medical Centre, Tokyo Medical University, between December 2016 and April 2018. Patient data was retrieved from a collected database and analyzed retrospectively. The indications for anatomical liver resection were determined based on the reserve liver function and were assessed using Makuuchi's criteria, which includes the preoperative measurements of ascites, serum bilirubin and ICG R15 (4).

Ethical products. The current study was performed in accordance with the Declaration of Helsinki, 1975 (as revised in 2008), and the regulations of the Japanese Ministry of Health, Labor and Welfare. The present study was approved by the research and ethics committee at Tokyo Medical University, Ibaraki Medical Center (permit no. 16-14). The patients who completed a follow-up examination were included in the current study. All study participants provided written informed consent.

$C T$. Contrast-enhanced CT images of $1 \mathrm{~mm}$ tumor tissue were obtained using a multi-detector CT (128 Columns; Brilliance iCT SP; Phillips Limited). The scan settings included a pitch of 17 , a $0.75 \mathrm{sec}$ scan time per rotation, a table speed of $12 \mathrm{~mm} /$ rotation and a detector configuration of 0.75 $916 \mathrm{~mm}$. A power injector was used to administer iopamidol $(600 \mathrm{mg} / \mathrm{kg}$ ), for $30 \mathrm{sec}$, through a $20-\mathrm{G}$ high-pressure intravenous catheter. Three sets of hepatic images were captured in succession, including images of the arterial, portal venous and hepatic venous phases. The arterial phase images were obtained $5 \mathrm{sec}$ after peak aortic enhancement. The portal venous phase images were obtained $70 \mathrm{sec}$ after the start of the injection. The hepatic venous phase was obtained $180 \mathrm{sec}$ after the start of the injection.

Evaluation utilizing a workstation (SV). The two-dimensional (2D) CT patient images were transferred to SV, and 3D images were generated. The liver, vessel network and tumor identification were evaluated, and the resectable liver region was determined.

Evaluation utilizing HSS. A basic algorithm incorporating the tumor dominance ratio was used. The resection region and ERLV of HSS was simulated using parameters (portal and hepatic vein, tumor location and total liver volume) gained from SV, which were also used to obtain the Stereolithography (STL) results (Fig. 1).

The relationship between the tumor and the tumor-associated region is presented in Fig. 2. The tumor is fed by part of the Grisson's pedicle, and the liver tumor metastasizes in the downstream direction through the portal vein. Therefore, the tumor-related region can be estimated based on the perfused region of the portal-vein (point P). Perfused regions are commonly estimated using a Voronoi diagram. Fig. 3 demonstrates the method of computing perfused regions with a Voronoi diagram. A Voronoi diagram divides space into several regions as presented in Fig. 3A. A set of points (called seeds) are specified and, for each seed, a corresponding region will be indicated that consists of all points closer to that seed than to any other. These corresponding regions are called Voronoi cells. In estimated perfused regions, a seed and Voronoi cell correspond to a point on a portal vein and a region perfumed by the portal point, respectively, as indicated in Fig. 3B. In Fig. 3B, portal-vein points (seeds) exist continuously, whereas seeds in the original Voronoi diagram are located apart. Therefore, the liver is divided into small regions, which equal the same number as the portal-vein points. The tumor domination ratio (TDR) of a portal-vein point $\mathrm{P}$ is defined as:

$$
\mathrm{TDR}=\frac{\text { (Volume of the region perfused of } \mathrm{P} \text { in the tumor) }}{\text { (Volume of the tumor) }} \times 100[\%]
$$

where the denominator and enumerator are presented in Fig. 3C. The TDR indicates how much the portal vein point feeds, and is affected by, the tumor. Following establishment of a tumor location, a complete tumor-associated region is estimated using the steps indicated in Fig. 4. Step I: Among the portal-vein points with a TDR $>0$ on a branch, select the point closest to the main stem as a cut point of the branch. In Fig. 4A, $\mathrm{Pi}(\mathrm{i}=1-6)$ are the portal-vein points with a TDR $>0$; $\mathrm{P} 1$ and $\mathrm{P} 4$ are selected as cut points on branches $\mathrm{B} 1$ and B2, respectively.

Step II: For each branch, the downstream part of the cut point is computed using the growing region (Fig. 4B). For all the portal-vein points of the downstream parts, the perfused regions are computed as presented in Fig. 4C.

Step III: The ideal resected region is obtained by the union of all perfused regions of all points on the downstream parts, as demonstrated in Fig. 4D.

Algorithm to calculate ERLV under maximum ERLV simulation. Although the $100 \%$ resected region is the best option to prevent cancer metastasis, it may not be obtainable for the majority of patients due to poor liver function. In pre-operative planning, surgeons usually determine the upper limit of the cut volume depending on the liver functionality of each patient. Therefore, it is important to identify an optimal resected region under the limitation of a cut-volume.

Estimating the optimal resected region is formulated as follows: $\mathrm{V}_{\max }$ is set at the maximum ERLV for each patient. This parameter must be provided by surgeons based on the result of the patients' pre-operative ICG R15. TDR low should be set at the lower limit of the TDR. The cut points with a tumor donation ratio of $\angle \mathrm{TDR}_{\text {low }}$ are not considered as this would result in a smaller resected region as $T D R_{\text {low }}$ is weakly associated with tumor and are omitted from the resected region. If $\mathrm{TDR}_{\text {low }}$ are not used, the algorithm sometimes selects the largest region with a very small TDR, and this optimization result is not reliable. Therefore, the parameter $\mathrm{TDR}_{\text {low }}$ is empirically set to $\sim 1 \%$ so that this does not occur.

As cut points, portal-vein points satisfying the following conditions are selected: The cut points are branch points, the thickness is larger than the pre-set thickness and the tumor donation ratio is $>\mathrm{TDR}_{\text {low }}$. $\mathrm{CP}$ should $=\left(\mathrm{c}_{\mathrm{i}} \mid 1 \leq \mathrm{i} \leq \mathrm{N}\right)$ for the set of cut points, where $\mathrm{N}$ is the total number of cut points. The optimization problem can be formulated as follows: 


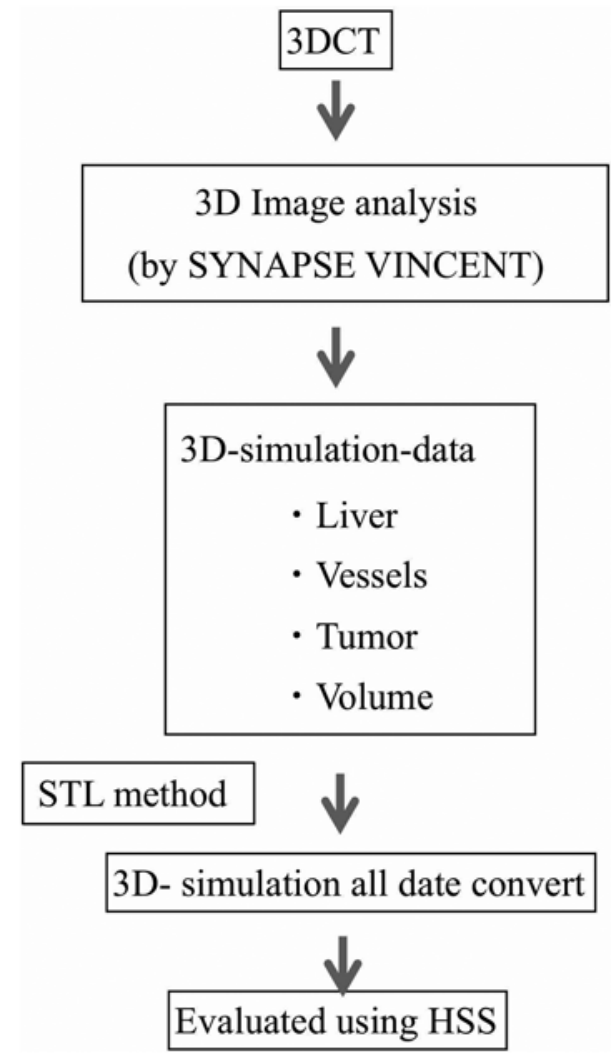

Figure 1. HSS obtained from SV results were used to convert to and obtain the STL method. HSS, Hariyama-Shimoda Soft; STL, stereolithography. SV, synapse Vincent.

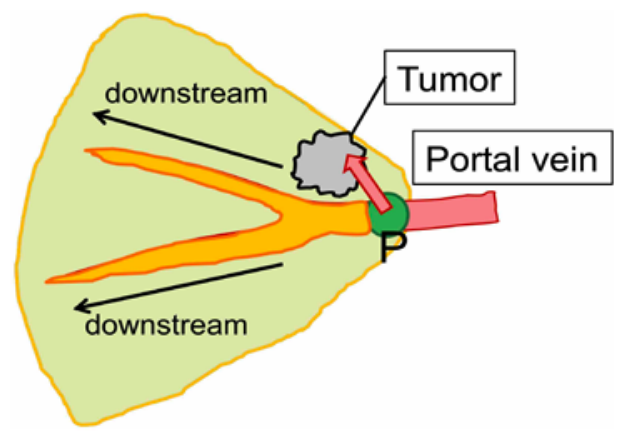

Figure 2. Relationship between tumor and tumor-associated regions.

maximize $\sum_{i}^{N} T_{i}$ under the conditions of $\sum_{i}^{N} V_{i} \leq V_{\max }$. By solving this optimization problem, the resected region maximizing the TDR is obtained. The resultant resected region is expected to be strongly associated with the tumor. Therefore, the proposed algorithm estimated the resected region that is most strongly associated with the tumor under the constraint of volume.

Considering the perfused regions of $\mathrm{P}_{1}$ and $\mathrm{P}_{2}$ as indicated in Fig. 4D, the volume and TDR of the perfused regions of $\mathrm{P}_{1}$ are $\mathrm{V}_{1}=200 \mathrm{cc}$ and $\mathrm{T}_{1}=70 \%$, respectively, and the volume and TDR of the perfused regions of $\mathrm{P}_{2}$ are $\mathrm{V}_{2}=150 \mathrm{cc}$ and $\mathrm{T}_{1}=30 \%$, respectively. For $\mathrm{V}_{\max }=150 \mathrm{cc}$, only the perfused region of $\mathrm{P}_{2}$ is selected as the resected region. For $\mathrm{V}_{\max }=200 \mathrm{cc}$, the perfused region of $\mathrm{P}_{1}$ is selected as the resected region. For $\mathrm{V}_{\max }>350 \mathrm{cc}$, both are selected as the resected regions.
Determining the proper $\mathrm{V}_{\max }$ is a critical issue in pre-operative simulation of resectable liver volume. If the estimated resected region is too large for the liver function, it would cause post-operative failure. Additionally, if the estimated resected region is too small, tumor metastasis would recur. Based on these considerations, $V_{\max }$ should be the maximum volume, while not causing post-operative failure. In the present study, $\mathrm{V}_{\max }$ was determined according to Makuuchi's criteria, as follows (Fig. 5):

$$
V \max =\left\{\begin{array}{c}
\left.\frac{2}{3} \times \operatorname{TLV} \text { (more than } 2 \text { sections }\right) \\
\frac{1}{3} \times \operatorname{TLV} \text { (sectinectomy) } \\
\frac{1}{6} \times(\text { subsegmentectomy })
\end{array}\right.
$$

where TLV is the total liver volume, and can be easily obtained via 3D simulation.

ERLV was compared with the actual resected liver volume (ARLV) using SV and HSS. Furthermore, HSS was revealed to be a useful simulation software for ARLV.

Operative procedure. The resection region, according to Couinaud's classification, was determined using staining techniques with intraoperative ultrasound (2). Under the guidance of ultra-sonographic imaging, approximately $5 \mathrm{ml}$ of indigo carmine dye (Indigo carmine Injection $20 \mathrm{mg} / 5 \mathrm{ml}$; Daiichi Sankyo UK Limited) was injected into a branch of the feeding portal vein. Additional branches were punctured, if necessary, depending on the location of the liver tumor. The liver parenchyma were divided using the clamp-crushing method or using an ultrasonic dissector. To control bleeding during parenchymal transection in this series, the intermittent Pringle maneuver was used (10 or 15 min clamping and $5 \mathrm{~min}$ release). After resection, abdominal drains were inserted into the liver cut surface, and a chest drain was inserted into the right pleural space, if necessary. Drainage tubes were removed when there was no visible bile leakage, the fluid bilirubin level was $<2.0 \mathrm{mg} / \mathrm{dl}$, and the amount of fluid collection was $<100 \mathrm{ml} /$ day from day 3 following surgery.

Statistical analysis. Statistical analyses were performed using the SPSS statistical software package (version 25.0; IBM Corp.). Medians were used to define each parameter. Categorical data were compared using the Kruskal-Wallis (non-parametric), as appropriate. Correlations were presented as scatterplots and were analyzed using Pearson's test. $\mathrm{P}<0.05$ was considered to indicate a statistically significant difference.

\section{Results}

Demography. A total of 15 patients were included in the current study, 3 female and 12 male, with a median age of 68.7 years (44.7-80.9 years). In the preoperative diagnosis, 12 of the 15 patients had HCC and 3 patients had colorectal liver metastasis requiring $\mathrm{AR}$ for tumor localization. A total of 6 out of 15 patients had hepatitis C, 4 patients had non-B non-C, 2 patients had hepatitis B, 2 patients had alcoholic liver disease and 1 patient had hepatitis B and C. The tumor locations were as follows: 4 patients in segment 7, 4 patients in segment 8,3 patients in segment 6,1 patient in segment 3 , 1 patient in segment 5, 1 patient in segments 5-6 and 1 patient in the anterior segment. Regarding the types of liver 
A

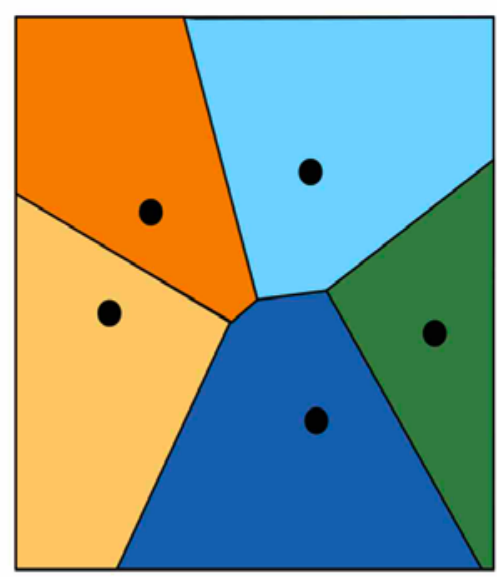

: : seed
B

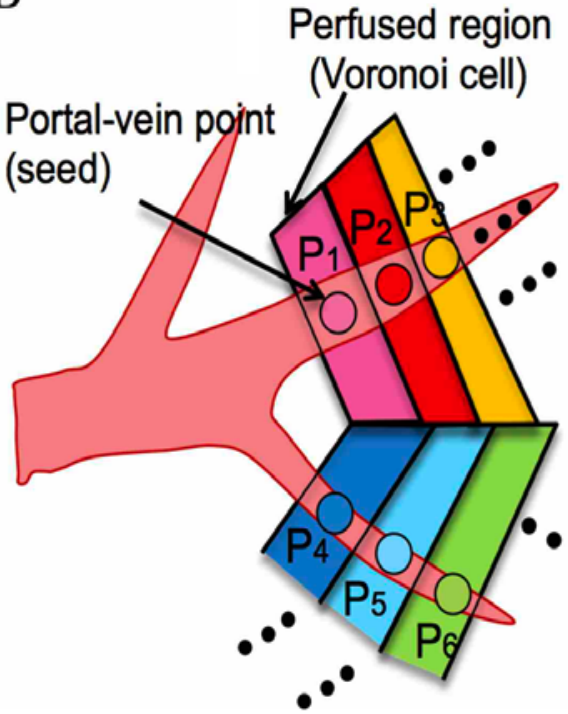

$\mathrm{C}$

Perfused region of $P$

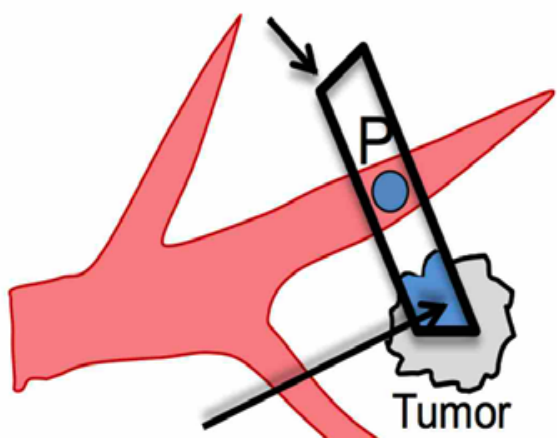

Perfused region of $R$ in the tumor

Figure 3. Estimation of the region perfused by portal-vein points based on the Voronoi diagram. (A) A Voronoi diagram divides space into several regions (B) A set of points (called seeds) are specified. For each seed, a corresponding region will be indicated, which consists of all points closer to that seed than to any other. In estimated perfused regions, a seed and Voronoi cell correspond to a point on a portal vein and a region perfumed by the portal point. (C) The denominator and enumerator are presented.

A

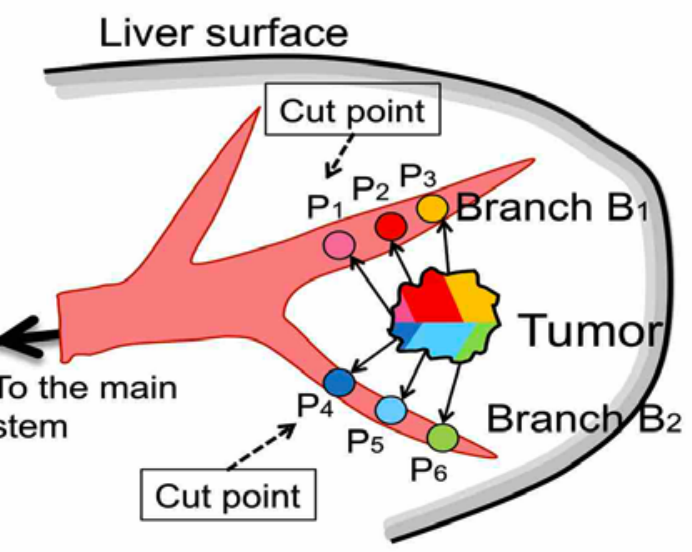

$\mathrm{C}$

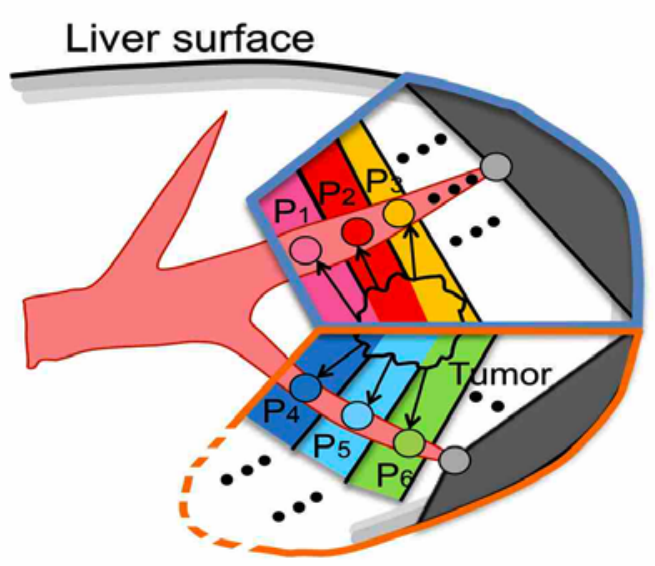

$\mathrm{B}$

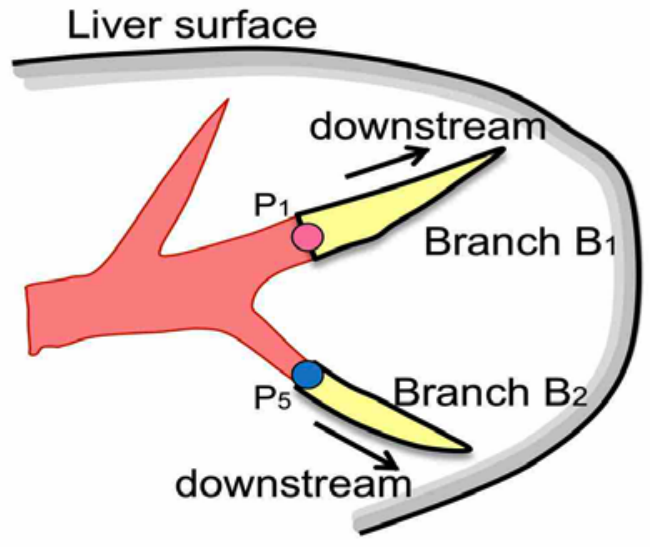

$\mathrm{D}$

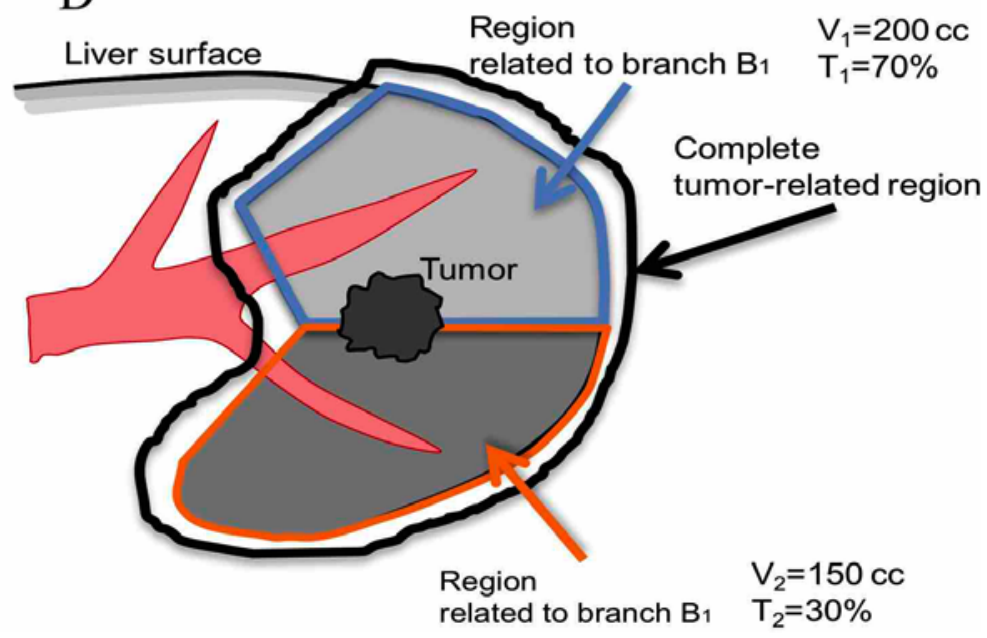

Figure 4. Estimation of the complete resected region using a basic algorithm. (A) Pi1-6 were the portal-vein points with a TDR $>0$. P1 and P4 were selected as cut points on branches B1 and B2. (B) For each branch, the downstream part of the cut point was computed using the growing region. (C) For all the portal-vein points of the downstream parts, the perfused regions were computed. (D) The ideal resected region was obtained by the union of all perfused regions of all points on the downstream parts. TDR, tumor domination ratio. 
Table I. Preoperative characteristics.

\begin{tabular}{|c|c|c|}
\hline Variable & Number of patients & Median (interquartile range) \\
\hline \multicolumn{3}{|l|}{ Sex } \\
\hline Male & 12 & \\
\hline Female & 3 & \\
\hline \multicolumn{3}{|c|}{ Preoperative diagnosis (cases) } \\
\hline $\mathrm{HCC}$ & 12 & - \\
\hline CRLM & 3 & - \\
\hline \multicolumn{3}{|c|}{ Underlying liver diseases (cases) } \\
\hline $\mathrm{HCV}$ & 6 & - \\
\hline NBNC & 4 & - \\
\hline HBV & 2 & - \\
\hline Alcoholic & 2 & - \\
\hline $\mathrm{HCV}$ and $\mathrm{HBV}$ & 1 & - \\
\hline \multicolumn{3}{|c|}{ Preoperative tumor location (cases) } \\
\hline Seg 7 & 4 & - \\
\hline Seg 8 & 4 & - \\
\hline Seg 6 & 3 & - \\
\hline Seg 3 & 1 & - \\
\hline Seg 5 & 1 & - \\
\hline Seg 5-6 & 1 & - \\
\hline Anterior sec & 1 & - \\
\hline ICG R15 (\%) & & $10.8(5.5-24.7)$ \\
\hline TLV (ml) & & $1253.0(881-1917)$ \\
\hline $\mathrm{V} \max (\mathrm{ml})$ & & $546.7(181.0-1089.3)$ \\
\hline$V \max =2 / 3 \times$ TLV (cases) & 7 & - \\
\hline$V \max =1 / 3 \times$ TLV (cases) & 4 & - \\
\hline$V \max =1 / 6 \times$ TLV (cases) & 4 & - \\
\hline
\end{tabular}

HCC, haeptocellular carcinoma; CRLM, colorectal carcinoma liver metastasis; HCV, hepatitis C; NBNC, none B and none C; HBV, hepatitis $\mathrm{B}$; seg, segment; sec, section; TLV, total liver volume; Vmax, maximum liver volume; ICG R15, idocyanine green retention test 15.

resection, 13 patients had subsegmentectomy and 2 patients had sectionectomy (Table I). The median operation time was $241 \mathrm{~min}$, the volume of blood loss was $320 \mathrm{ml}$ and the Pringle maneuver time was $80 \mathrm{~min}$. Histopathologically, 11 patients had HCC, 3 patients had colorectal carcinoma with liver metastasis and 1 patient had intrahepatic cholangiocarcinoma. The median tumor size was $3.5 \mathrm{~cm}$ (range: $1.2-6.7 \mathrm{~cm}$; Table II). No operation-associated deaths occurred in these patients, no tumor recurrence occurred and no patients suffered from cancer-associated mortality during the study period.

Vmax evaluation. The median ICG R15 was $10.8 \%$ (range: $5.5-24.7 \%$ ), the median TLV was $1253.0 \mathrm{ml}$ (range: $881-1917 \mathrm{ml}$ ) and the median $\mathrm{V} \max$ was $546.7 \mathrm{ml}$ (range: 181.0-1089.3 ml). The number of patients with $\mathrm{V} \max =2 / 3 \times \mathrm{TLV}$ was 7 , with $\mathrm{V} \max =1 / 3 \mathrm{x}$ TLV case was 4 and with $V$ max $=1 / 6 x$ TLV was 4 .

Typical case. A case analysis using HSS is indicated below. To demonstrate the performance of the proposed method, the results of SV and HSS were compared. Fig. 5 illustrates how the proposed algorithm works. Fig. 6A visualizes the TDR. The region near the tumor exhibited a high TDR. A large grey region is observed, for which the TDR is $<0-1 \%$. Although this grey region exhibited a much weaker relationship than other regions, it occupies a larger volume. If the volume of the complete tumor-related region is too large for the patient, the grey region would be omitted from the resected region. The planning result in SV is indicated in Fig. 6B. The surgeon obtained this result manually without TDR. As indicated in Fig. 6A and $\mathrm{B}$, the surgeon omits the region with a TDR of $<1 \%$.

The planning result obtained automatically by HSS is demonstrated in Fig. $6 \mathrm{C}$. In the present study, $\mathrm{TDR}_{\text {low }}$ is set to $1 \%$ and $\mathrm{V}_{\max }$ is determined to be almost the same as when using SV. These results demonstrated that the proposed algorithm can automatically produce the same result as a skilled surgeon.

Comparison of ERLV and ARLV for HSS and SV. The median estimated maximum resectable liver volume was determined according to Makuuchi's criteria. The median ERLVs for SV and HSS were $147.5 \mathrm{ml}(67-267 \mathrm{ml})$ and $184.0 \mathrm{ml}(43-457 \mathrm{ml})$, respectively, with no significant variance between the groups 
Table. II. Operative and histological findings.

\begin{tabular}{lcc}
\hline Variable & Number of patients & Median (interquartile range) \\
\hline Type of liver resection & 13 & - \\
Subsegmentectomy & 2 & - \\
Sectionectomy & - & $241(165-438)$ \\
Operating time (min) & - & $80(15-210)$ \\
Pringle time (min) & - & $320(40-1294)$ \\
Blood loss (ml) & & - \\
Histological diagnosis & 11 & - \\
HCC & 3 & - \\
CRLM & 1 & $3.5(1.2-6.7)$ \\
ICC & - &
\end{tabular}

HCC, hepatocellular carcinoma; CRLM, colorectal carcinoma liver metastasis; ICC, intrahepatic cholangiocarcinoma.

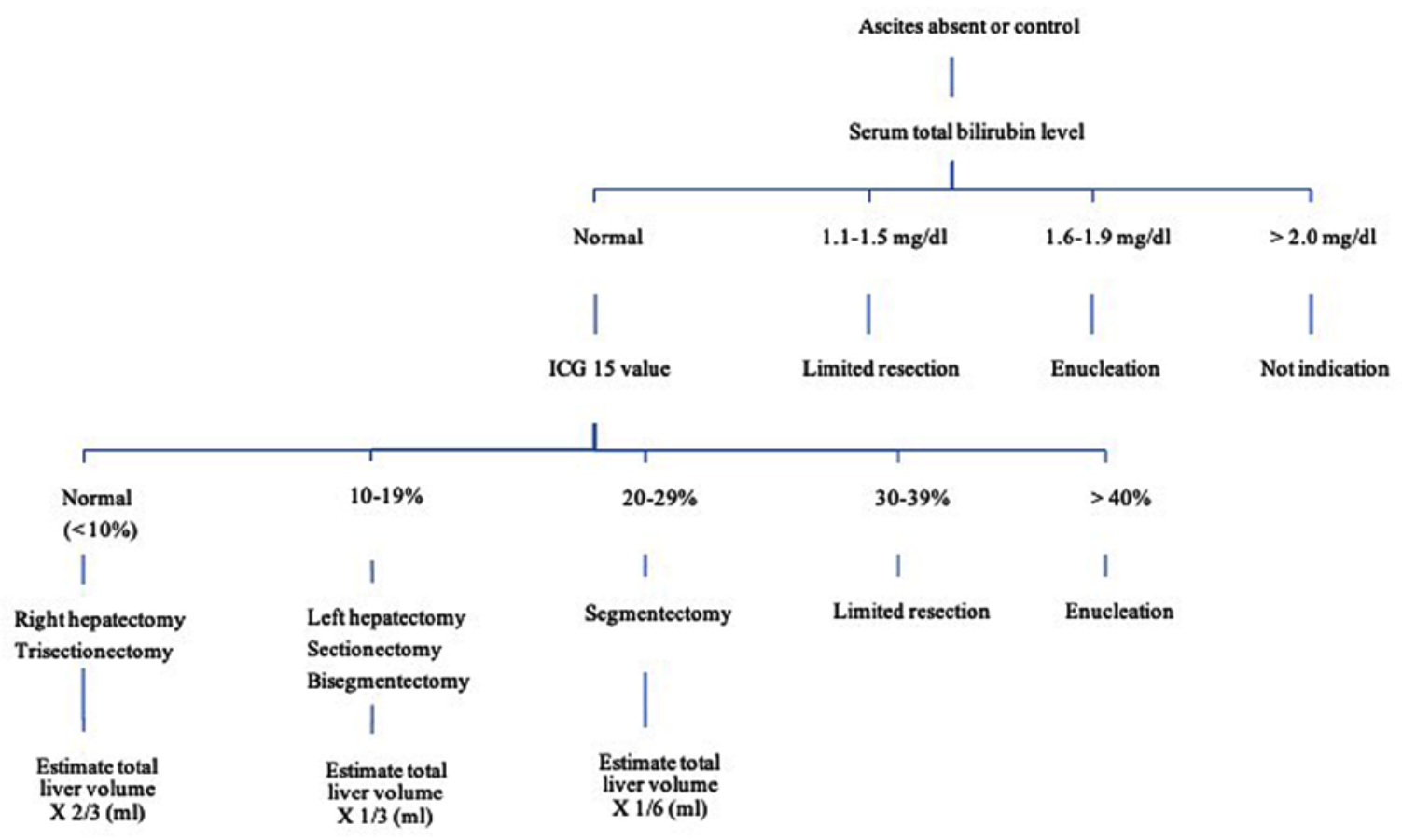

Figure 5. Estimation of the maximum resectable liver volume according to Makuuchi's criteria.

( $\mathrm{p}=0.15)$. The median ARLV was $134.0 \mathrm{~g}(60-320 \mathrm{~g})$ and no significant variance was demonstrated in ERLV using SV or HSS ( $\mathrm{p}=0.28$; Fig. 7).

The correlation between ERLV and ARLV among SV and HSS were as follows, and statistically significant correlations were seen: SV ERLV $(\mathrm{ml})=1.139 \mathrm{X} \operatorname{HSS} \operatorname{ERLV}(\mathrm{ml})+$ $30.779(\mathrm{r}=0.763 \mathrm{p}=0.001$; Fig. 8A). ARLV $(\mathrm{g})=1.252 \mathrm{X} \mathrm{SV}$ $\operatorname{ERLV}(\mathrm{ml})-42.676(\mathrm{r}=0.909 \mathrm{p}=0.0001$; Fig. 8B). ARLV $(\mathrm{g})=$ 0.799 X HSS ERLV $(\mathrm{ml})+80.611(\mathrm{r}=0.725 \mathrm{p}=0.003$; Fig. $8 \mathrm{C})$.

\section{Discussion}

The purpose of visual liver imaging is to identify or deliver a qualitative diagnosis through $2 \mathrm{D}$ imaging, using CT or ultrasound. However, due to the liver being a parenchymal organ, it is impossible to estimate the precise resection volume of the liver, with consideration of the portal veins and vessel networks, statically, through 2D CT imaging. Recent advancements in CT imaging have led to a variety of 3D CT simulation software platforms since 2000 , and steric imaging of the liver has become possible $(1,5,6)$. These advancements have allowed easier understanding and identification of tumor location, estimation of the resection region and liver volume, shortened surgery time and decreased blood loss $(1,7)$. These advancements have also contributed to the prevention of post-operative liver failure, and currently, 3D CT imaging has become an essential tool in the planning and performance of liver resection $(1,7,8)$. 

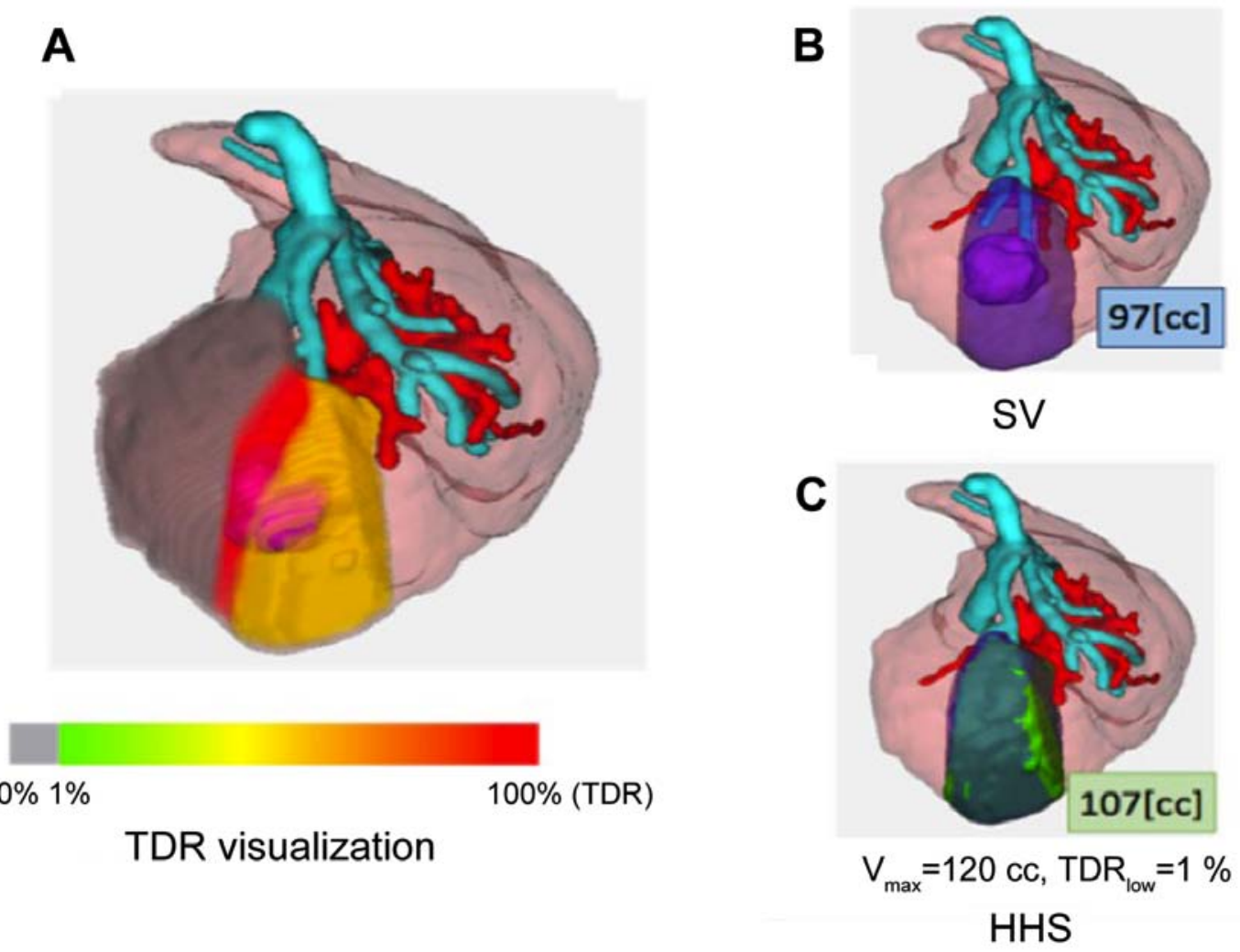

Figure 6. TDR visualization and the results of HSS were compared with SV. (A) Visualization of the 100\% TDR. (B) Planning result of SV (none automatic). (C) Planning result obtained by HSS (automatic), omitting regions with a TDR of $<1 \%$ (gray area) from $100 \%$ TDR. Responsible portal vein is presented in the gray area (Black arrow). TDR, tumor domination ratio; HSS, Hariyama-Shimoda Soft; SV, synapse Vincent.

Liver failure is a critical complication following hepatectomy. Makuuchi, et al proposed a standard for an acceptable hepatectomy range using ICG R15 during the 1990s (4,9). These criteria have been accepted at many institutions in Japan, and post-operative liver failure has been reduced using these, improving the safety of liver resection $(2,10)$. The criteria utilize Couinaud's anatomical liver segmentation (11). Segmentation of the liver by Couinaud is based on the location of hepatic veins and for subsegmentectomy, the boundary is set based on the third portal branch, and is therefore very broad (11). It is a common understanding that Couinaud's classification is not applicable for all cases due to the variations in branching or in the network of veins, which differ with every patient. However, the introduction of 3D image simulation has allowed steric imaging of portal veins, other vessel networks and portal branches in detail, and has allowed precise simulation of sectionectomy or subsegmentectomy. Additionally, it has allowed detailed anatomical classification of liver sections and subsegments.

Liver subsegmentectomy, proposed by Makuuchi et al, utilizes the portal vein puncture staining method using ultrasound during the operation, and is a method that minimizes the liver resected volume while improving the complete cure rate $(2,10,12)$. This operational method bases its resection region on Couinaud's subsegmentation, has improved the complete cure rate and has reduced post-operative liver failure. However, evaluating the ERLV through 2D CT is difficult, and a comparison of ARLVs has not been evaluated. SV allows detailed identification of subsegments due to its identification

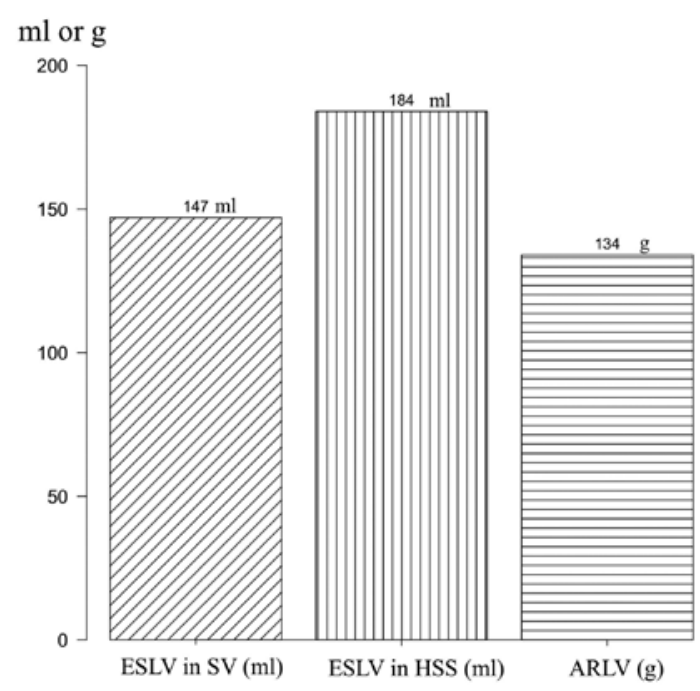

Figure 7. Median ERLV for SV and HSS, and the median ARLV. ERLV, estimated resectable liver volume; SV, synapse Vincent; HSS, Hariyama-Shimoda Soft; ARLV, actual resected liver volume.

of dominant portal branches in the tumor. Takamoto et al (1) demonstrated the efficacy of SV for pre-operative simulation and that there was no significant variance between ERLV and ARLV, concluding that SV was useful as an index for anatomical liver resection. However, SV requires the surgeon to manually identify all the portal veins feeding into the tumor to determine the resection region. Therefore, it is difficult 

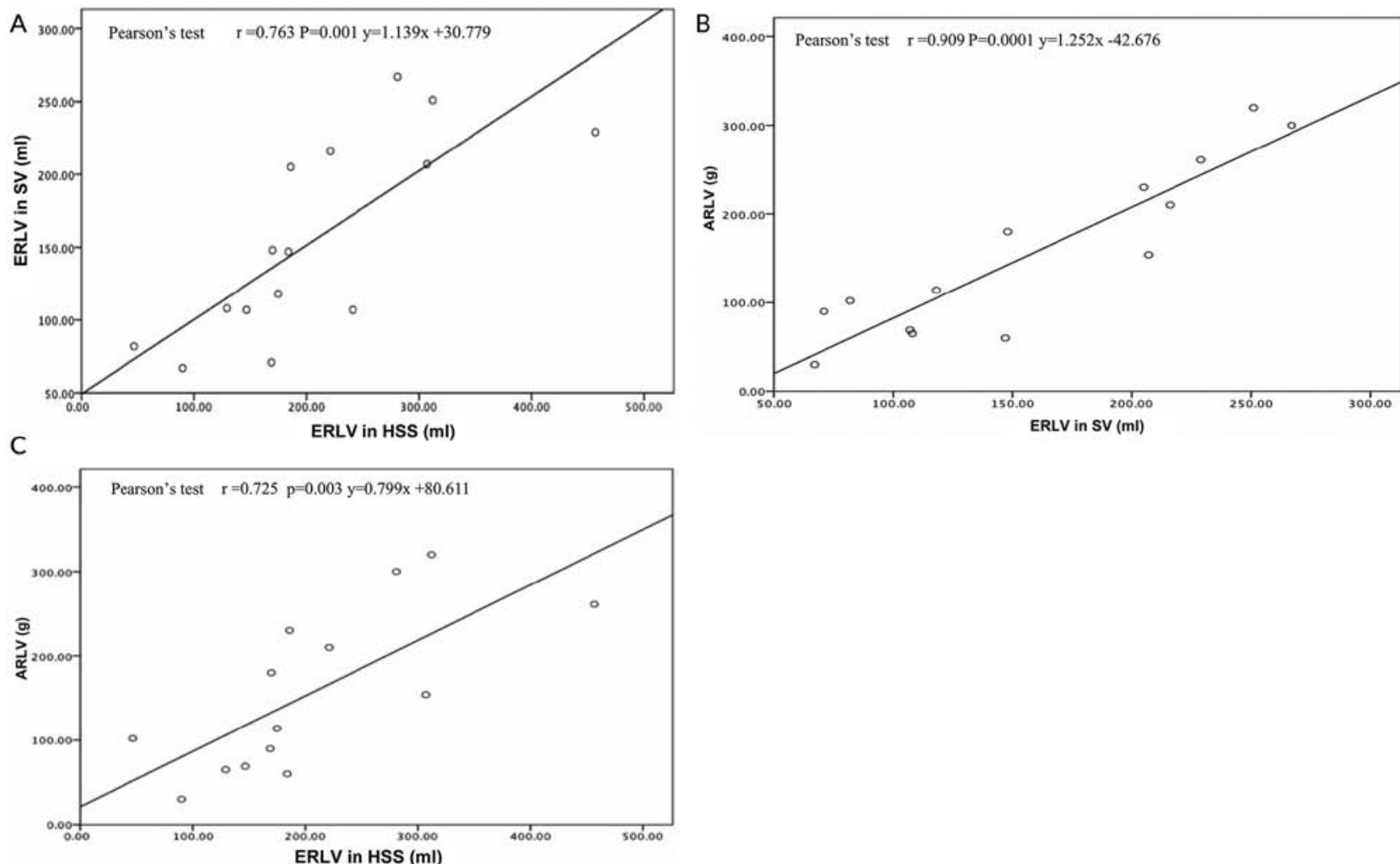

Figure 8. Correlation between ERLV and ARLV among SV and HSS. (A) Comparison of ERLV and HSS or SV. (B) Comparison of ARLV and ERLV in SV. (C) Comparison of ARLV and ERLV in HSS. ERLV, estimated resectable liver volume; ARLV, actual resected liver volume HSS, Hariyama-Shimoda Soft; SV, synapse Vincent.

to conclude whether all the portal veins are identified using this method. Additionally, the preoperative status of the liver (chronic hepatitis, fatty liver or cirrhosis) is typically ignored when determining the ERLV. Therefore, it is difficult to determine if the resection region would be too large or whether the operation would be sufficient to prevent recurrence.

In this software, a function that automatically $100 \%$ identifies the tumor dominant portal vein from the tumor perspective and estimates the maximum resection volume, based on Makuuchi's criteria, was incorporated into HSS. In the current study, the ERLV via HSS and SV was within the maximum ERLV, based on Makuuchi's criteria, and there was no statistically significant difference between the ARLVs. The novel HSS incorporates the function to identify portal vein branches dominating the tumor automatically, and ERLV enables the resection volume to be minimal, improving the complete cure rate of hepatic cancer. Additionally, HSS can identify the maximum resection volume to prevent liver failure. HSS (automated) has proven its efficacy as a $3 \mathrm{D}$ simulation software compared with SV (manual).

In the present study, no cases of SV and HSS exceeding the ERLV derived from Makuuchi's criteria were observed. These results indicated the possibility that Makuuchi's criteria overestimated the resection region. This further indicated that the acceptable resection region identified through these criteria was actually the maximum ERLV that does not induce post-operative liver failure. Therefore, to set a more suitable resection region, and to prevent recurrence, it is necessary to utilize 3D simulation software and determine regions for sectionectomy and subsegmentectomy, thereby replacing the current Couinaud segmentation. Using this method, surgeons could identify the optimal section or subsegment region with the minimum resection liver volume that enables the maximum possibility of a complete cure. To realize the optimal hepatectomy, it is necessary to resect the region identified through the $3 \mathrm{D}$ simulation software accurately, and a new real-time operational navigation system needs to be developed to utilize the 3D simulation images accurately.

This study had a limitation that the sample size is small, and further research will be performed to compare SV and HSS in more detail, such as mobility and mortality.

In conclusion, the HSS functions to identify a tumors dominant portal vein and has been proven effective as a new factor to be incorporated into simulation software. The acceptable resection region identified by the Makuuchi's criteria was the estimate of maximum resection volume that does not include liver failure. The resection region identified via HSS was the maximum resectable volume that prevents recurrence. Future studies should include an increased sample size and include thorough follow-ups on the prognosis of each patient.

\section{Acknowledgements}

The authors would like to thank Mr. Hitoshi Iizumi and Mr. Gen Arai (Department of Radiology, Tokyo Medical University, Ibaraki, Medical Center, radiological technician) for their assistance with STL conversion and CT creation. The current study (Volume 20, Supplement 2, S173-S846) was presented at the 13th World Congress of the International Hepato-Pancreato-Biliary Associations conference in Geneva, Switzerland on the 4th-7th September 2018. 


\section{Funding}

This study was supported by MEXT KAKENHI grant number 24500242

\section{Availability of data and materials}

The datasets used and/or analyzed in the present study are available from the corresponding author on reasonable request.

\section{Authors' contributions}

MS and MH analyzed and interpreted CT and 3D simulation data. MH performed and analyzed 3D simulation data. MS, YO and SS performed liver surgery. MS and SS wrote the manuscript.

\section{Ethics approval and consent to participate}

The current study was performed in accordance with the Declaration of Helsinki, 1975 (as revised in 2008), and the regulations of the Japanese Ministry of Health, Labour and Welfare. The present study was approved by the Research and Ethics Committee of Tokyo Medical University, Ibaraki Medical Center (permit no. 16-14).

\section{Patient consent for publication}

Not applicable.

\section{Competing interests}

The authors declare that they have no competing interests.

\section{References}

1. Takamoto T, Hashimoto T, Ogata S, Inoue K, Maruyama Y, Miyazaki A and Makuuchi M: Planning of anatomical liver segmentectomy and subsegmentectomy with 3-dimensional simulation software. Am J Surg 206: 530-538, 2013.
2. Makuuchi M, Hasegawa $\mathrm{H}$ and Yamazaki S: Ultrasonically guided subsegmentectomy. Surg Gynecol Obstet 161: 346-350, 1985.

3. Hariyama T, Sandborn PAM, Watanabe M and Wu MC: High-accuracy range-sensing system based on FMCW using low-cost VCSEL. Opt Express 26: 9285-9297, 2018.

4. Miyagawa S, Makuuchi M, Kawasaki S and Kakazu T: Criteria for safe hepatic resection. Am J Surg 169: 589-594, 1995.

5. Selle D, Preim B, Schenk A and Peitgen HO: Analysis of vasculature for liver surgical planning. IEEE Trans Med Imaging 21: 1344-1357, 2002.

6. Saito S, Yamanaka J, Miura K, Nakao N, Nagao T, Sugimoto T, Hirano T, Kuroda N, Iimuro Y and Fujimoto J: A novel 3D hepatectomy simulation based on liver circulation: Application to liver resection and transplantation. Hepatology 41: 1297-1304, 2005.

7. Nakayama K, Oshiro Y, Miyamoto R, Kohno K, Fukunaga K and Ohkohchi N: The effect of three-dimensional preoperative simulation on liver surgery. World J Surg 41: 1840-1847, 2017.

8. Lamadé W, Glombitza G, Fischer L, Chiu P, Cárdenas CE Sr, Thorn M, Meinzer HP, Grenacher L, Bauer H, Lehnert T and Herfarth C: The impact of 3-dimensional reconstructions on operation planning in liver surgery. Arch Surg 135: 1256-1261, 2000.

9. Makuuchi M, Kosuge T, Takayama T, Yamazaki S, Kakazu T, Miyagawa S and Kawasaki S: Surgery for small liver cancers. Semin Surg Oncol 9: 298-304, 1993.

10. Makuuchi M, Hasegawa $\mathrm{H}$ and Yamazaki S: Intraoperative ultrasonic examination for hepatectomy. Ultrasound Med Biol (Suppl 2): 493-497, 1983.

11. Couinaud C: Liver lobes and segments: Notes on the anatomical architecture and surgery of the liver. Presse Med 62: 709-712, 1954 (In French).

12. Shimoda M, Tago K, Kato M, Aoki T and Kubota K: Prognostic factors after curative resection for single-lesion hepatocellular carcinoma in patients with normal liver function: A historical cohort study. Dig Surg 34: 281-288, 2017.

This work is licensed under a Creative Commons Attribution-NonCommercial-NoDerivatives 4.0 International (CC BY-NC-ND 4.0) License. 